\title{
SPATIAL AND TEMPORAL VARIATIONS OF Ca II K LINE PROFILE SHAPES \\ IN DIFFERENT STRUCTURES OF THE SOLAR CHROMOSPHERE. I. FEATURES OF INDIVIDUAL PROFILES
}

\section{I.P. Turova}

Institute of Solar-Terrestrial Physics SB RAS, Irkutsk, Russia, turova@iszf.irk.ru

\section{S.A. Grigoryeva}

Institute of Solar-Terrestrial Physics SB RAS,

Irkutsk,Russia,sgrig@iszf.irk.ru

\author{
O.A. Ozhogina \\ Institute of Solar-Terrestrial Physics SB RAS, \\ Irkutsk,Russia,ozhog@iszf.irk.ru
}

\begin{abstract}
We have studied Ca II K line profiles, using two time series of spectrograms taken in two regions near the solar disk center. In each of the regions, the spectrograph slit cut out several areas of the quiet region and a plage. For the selected chromospheric structures, we have derived $\mathrm{K}$ line profiles and have defined a number of parameters that characterize the spatial and temporal variations of the profiles. The analysis of profile shapes in different structures belonging to the same moment of time has shown that there are structures whose profiles differ only slightly from each other in the photosphere, but differ dramatically in the chromosphere. The structures begin to differ from the level of formation of $\mathrm{K}_{1}$ and continue to differ further in the chromosphere. There are, however, structures which begin to differ at the level of the photosphere and continue to differ in the chromosphere. The difference between profile shapes in different structures is likely to be associated both with different thermodynamic conditions and with different magnetic field topology at a
\end{abstract}

given point at a given time. We have examined temporal variations of the $\mathrm{Ca}$ II K line profiles in structural chromospheric elements, which are caused by the process of $\mathrm{K}_{2 \mathrm{v}}$ grains. In most of the studied areas of the chromospheric structures, the brightening of the $\mathrm{K}_{2 \mathrm{v}}$ peak develops according to the "common" scenario: at the time of maximum brightness, the line shifts toward the red side. There are, however, cases when the brightening of the $K_{2 v}$ peak occurs with a shift of the line to the violet side or with no shift at all. We have constructed scatter plots for some pairs of profile parameters related to intensities at characteristic points of the profile and their shifts. A correlation has been found between intensities in the center and wings of the $\mathrm{K}$ line. The correlation between shifts of the $K_{2 v}$ and $K_{2 r}$ peaks is very weak or completely absent.

Keywords: chromosphere, Ca II K profiles, $\mathrm{K}_{2 \mathrm{v}}$ grains.

\section{INTRODUCTION}

Due to the development of observational facilities and theoretical methods, a wealth of knowledge about the solar chromosphere has been obtained, but a number of issues related to the physical processes occurring in this layer need to be further elaborated. Of interest here are the results that can confirm the previously obtained results and add confidence in the reliability of the latter.

This paper is a sequel to [Grigoryeva et al., 2016]. The authors have examined Ca II lines, using observations of two regions located at the base of a coronal hole. Each of these regions contains an area of the quiet chromosphere with bright and dark structures and a plage.

Our purpose here is to examine some features of spatial and temporal variations of $\mathrm{Ca}$ II $\mathrm{K}$ line profiles in various chromospheric structures.

\section{OBSERVATIONS} AND THEIR PROCESSING

Spectrograms in the Ca II lines were obtained with the Horizontal Solar Telescope of the Sayan Solar Observatory on August 3, 2005. Spectra were recorded with the Princeton Instrument 2048x2048 ma-
trix/(EEV/CCD) in the IV order. The dispersion is $0.0023 \mathrm{~nm} / \mathrm{pixel}$, the spatial size is $0.45^{\prime \prime} / \mathrm{pixel}$. The spatial resolution limited by visibility conditions is $\sim 2 "$. The exposure time is $2.5 \mathrm{~s}$; the cadence is $30 \mathrm{~s}$. The size of the solar surface limited by the spectrograph slit height is $\approx 200^{\prime \prime}$. All captured frames are corrected for the dark current and flat field.

We have selected two time series of $\approx 25 \mathrm{~min}$ spectrograms for this study. Each series contains 45 frames. The frames represent solar regions with coordinates S25W17 and S25W12 at the base of an extensive coronal hole. The S25W12 region has a bright coronal point. The frames of each of these regions contain areas of the quiet chromosphere and one plage.

For each spatial pixel in each frame, we have constructed Ca II K profiles. The total number of the profiles in both the series is $\approx 41000$.

Observation details and identification of chromospheric structures are described by Grigoryeva et al. [2016].

For our study, we have distinguished chromospheric structures notated as follows:

- " $n$ " is a bright network structure ("network");

- "ne" is an enhanced network;

- "c" is a dark internetwork structure ("internetwork"); 
- "b" is a plage;

- " $p$ " is a structure with low brightness, which is observed in frames of one of the two series;

- "f" are areas on boundaries of the selected structures;

- " $\mathrm{x}$ " is a structure with intermediate brightness, which does not belong to the above structures.

All the structures, except for the plage, are structural chromospheric network elements. The total number of the structures is 40 for S25W12 and 30 for S25W17.

The " $n$ ", "f", and "c" structures are similar to those identified by Reardon et al., [2008] from IBIS observations, where " $\mathrm{f}$ " areas correspond to fibrils; and " $\mathrm{x}$ ", to unclassified areas, which the authors do not analyze (see Figure 1 in [Reardon et al., 2008]). These structures have been identified from the time-averaged intensity $I_{\mathrm{K} 3}$ along the spectrograph slit $\left(I_{\mathrm{K} 3}\right.$ is a minimum central intensity of the $\mathrm{K}$ line). The distribution of $I_{\mathrm{K} 3}$ for the two regions is shown in Figure 1.

Most of the recent results on the $\mathrm{Ca}$ II lines are based on filter observations. Despite the undoubted value of such observations providing two-dimensional images, they have serious limitations due to filter bandwidth. The most up-to-date calcium filters have FWHM of $0.3 \AA$, while the contribution of the chromosphere to the $\mathrm{Ca}$ II $\mathrm{H}$ and $\mathrm{K}$ lines is restricted to narrow line cores less than $0.2 \AA$ wide [Reardon et al., 2009]. An exception is the CHROMIS instrument [Löfdahl et al., 2018], put into operation at the Swedish 1-m Solar Telescope (SST) in August 2016.
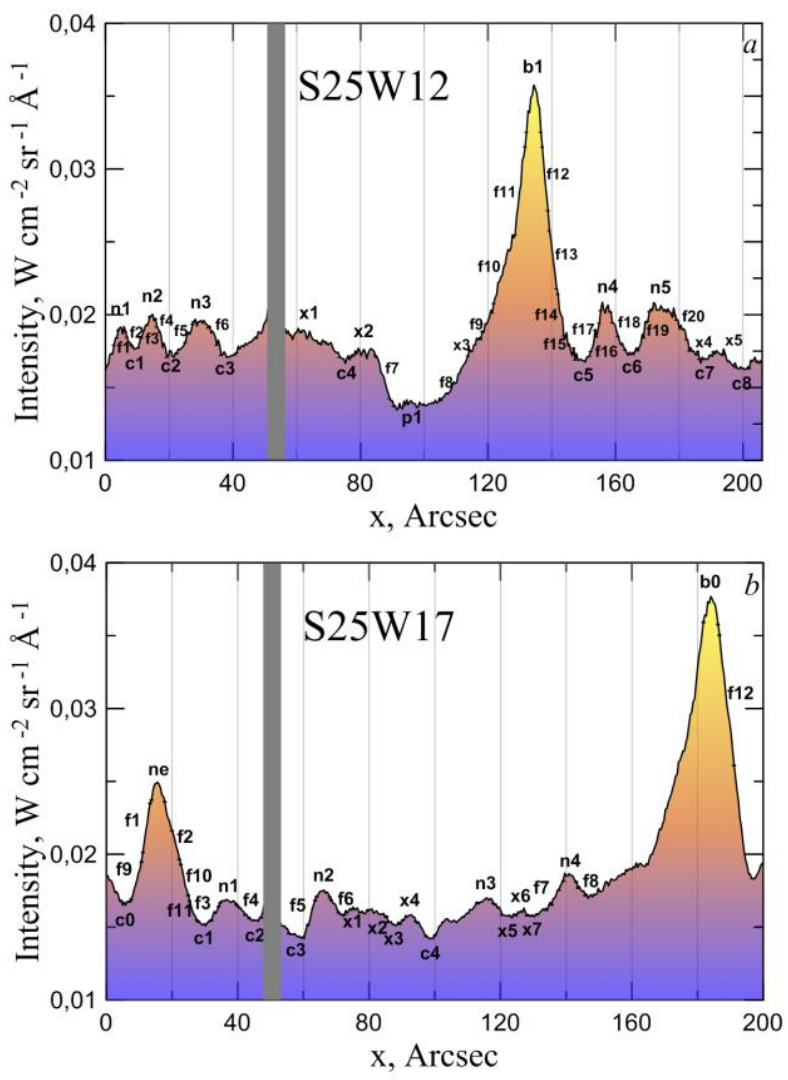

Figure 1. Time-averaged intensity $I_{\mathrm{K} 3}$ along the spectrograph slit: S25W12 (a); S25W17 (b). The selected chromospheric structures are denoted by letters and numbers. The gray vertical strip is a thread stretched across the spectrograph slit
The filter bandpass is currently $\sim 0.13 \AA$ and will be reduced to $0.08 \AA$ in 2019 . Filters with a much greater FWHM ( $3 \AA$ ) are very often used. Such observations provide mixed information about a large layer of the solar atmosphere - from the photosphere to the chromosphere. Spectroscopic observations are free of such restrictions, despite them having their own, related in particular to the image jitter on the spectrograph slit. A guiding system and a system of compensation for solar rotation in observation of time series make it possible to keep an image on the slit, but cannot cope with a small jitter caused by atmospheric turbulence. The jitter can be partially compensated (in one coordinate) using postprocessing methods. In particular, we superimpose consecutive frames according to the maximum correlation coefficient.

We have determined some quantitative parameters of Ca II K line profiles which can characterize spatial and temporal variations of the profiles:

- $I_{\mathrm{K} 3}$ is a minimum central intensity of the $\mathrm{K}$ line;

- $I_{\mathrm{K} 2 \mathrm{v}}, I_{\mathrm{K} 2 \mathrm{r}}$ are intensities of the violet and red emission peaks respectively;

- $I_{\mathrm{K} 2 \mathrm{v}} / I_{\mathrm{K} 2 \mathrm{r}}$ is the ratio of peak intensities;

- $\Delta \lambda_{\mathrm{K} 3}$ is a shift of the $\mathrm{K}_{3}$ minimum along the wavelength axis relative to the nominal line center;

- $\Delta \lambda_{\mathrm{K} 2 \mathrm{v}}, \Delta \lambda_{\mathrm{K} 2 \mathrm{r}}$ are shifts of the $\mathrm{K}_{2 \mathrm{v}}$ and $\mathrm{K}_{2 \mathrm{r}}$ peaks relative to the nominal line center;

- $\Delta \lambda_{\mathrm{K} 2 \mathrm{r}}-\Delta \lambda_{\mathrm{K} 2 \mathrm{v}}$ is the distance between the $\mathrm{K}_{2 \mathrm{r}}$ and $\mathrm{K}_{2 \mathrm{v}}$ peaks;

- $\Delta \lambda_{\mathrm{K} 2 \text { av }}=\left(\Delta \lambda_{\mathrm{K} 2 \mathrm{r}}+\Delta \lambda_{\mathrm{K} 2 \mathrm{v}}\right) / 2$ is the average shift of the $K_{2 v}$ and $K_{2 r}$ peaks relative to the nominal line center.

We have developed an IDL code to automatically determine the above listed parameters of the line profiles. During the computation, we faced a problem similar to that described in [Bjørgen et al., 2017]. There were very unusual line profiles, e.g., with three $\mathrm{K}_{2}$ peaks instead of two, with the absence of one or both $\mathrm{K}_{2}$ peaks or with very large shifts of $\mathrm{K}_{2}$ peaks and $\mathrm{K}_{3}$ minimum along the wavelength. In some of these cases, the code gave an incorrect identification of the parameters and we had to determine the parameters manually. We do not present data on variations of the profile parameters in $\mathrm{K}_{1 \mathrm{v}}$ and $\mathrm{K}_{1 \mathrm{r}}$. The $\mathrm{K}_{1 \mathrm{r}}$ minima is poorly accessible to computer processing because the $\mathrm{K}_{1 \mathrm{r}}$ minimum is very often absent. Hence, data on the intensity of this feature and its location are extremely noisy and of little real worth. The $\mathrm{K}_{1 \mathrm{v}}$ minimum is noisy for the same reason, although to a lesser extent.

\section{SPATIAL AND TEMPORAL VARIATIONS OF Ca II K LINE PROFILES}

It is known that the $\mathrm{Ca}$ II $\mathrm{H}$ and $\mathrm{K}$ line profiles can considerably vary in space and time. This phenomenon has been studied for many years. The obtained results have allowed significant progress in the study of the solar chromosphere (see, among others, [Pasachoff, 1970; Bappu, Sivaraman, 1971; Liu, Skumanich, 1974; Rezaei et al., 2008; Beck et al., 2008; Beck et al., 2009; Bjørgen et al., 2017]).

In our previous work [Grigoryeva et al., 2016], we have shown a large number of individual $\mathrm{Ca}$ II $\mathrm{K}$ pro- 
files observed in the chromospheric structures we selected (see Figure 4-7 [Grigoryeva et al., 2016]). We examined the profiles in a wavelength range $\Delta \lambda= \pm 0.5 \AA$.

In this paper, to study spatial and temporal variations of the profiles, we have analyzed the dynamics of the intensity in the $\mathrm{Ca}$ II $\mathrm{K}$ line in a longer wavelength range: $\Delta \lambda= \pm 2.0 \AA$.

\subsection{Spatial variations of profiles}

To study spatial variations of the Ca II K profiles, we have compared individual profiles observed at the same moment of time in different chromospheric structures. We noticed that some structures with extremely different physical conditions (see, e.g., 1D FAL models [Fontenla et al., 2009]) exhibit identical intensity variations in the line wing. Conversely, the structure of the same type may differ in intensity throughout the range $\Delta \lambda= \pm(0.2-2.0) \AA$. For the comparison, we selected profiles in which such ratios of intensities were most pronounced. We compared profiles related to the locations near the centers of the selected structures; each of the profiles had a minimum shift of $I_{\mathrm{K} 3}$ from the line center. This approach allows a more correct comparison of intensity variations in different structures.

Figure 2, $a-c$ shows profiles from one of the frames of the time series of S25W12. All the profiles are given in the same intensity and wavelength scales.

Figure 2, $a$ presents the K-line profiles associated with plage "b1", internetwork "c2", and area with low brightness "p1". Particularly notable is the significant difference between the profiles in the line core and $\mathrm{K}_{2}$ features. The profiles differ not only in central intensity, but also in shape. At the same time, in the line wing at $\Delta \lambda \approx \pm(0.5-2.0) \AA$, intensities in all the three structures are almost identical.

Figure 2, $b$ displays the profiles which are observed in "c5", "n4", and "n5". At the line core in the $\mathrm{K}_{2}$ features, profiles of these structures also differ from each other. It is interesting that in the wavelength range $\Delta \lambda \approx$ $\approx \pm(0.5-2.0) \AA$ intensities in internetwork "c5" and network " $\mathrm{n} 4$ " are similar. Intensities in the structures of the same type "n4" and " $\mathrm{n} 5$ " differ; the difference holds over the entire wavelength range $\Delta \lambda \approx \pm(0.5-2.0) \AA$.

Figure 2, c presents the profiles related to "c2", " $\mathrm{3}$ ", and "x1". Intensities of the " $\mathrm{n} 3$ " and "x1" structures at the line core are similar, but differ from the "c2" intensity. In the line wing in $\Delta \lambda \approx \pm(0.2 \div 2.0) \AA$, the three structures also differ significantly. The brightest wing is provided by "n3"; the darkest, by " $\mathrm{x} 1$ ". The line intensity in "c2" in this part of the line wing is located in an intermediate position. We do not give Figures for S25W17 because such behavior of intensities in the Ca II $\mathrm{K}$ line core and wing also persists in a number of the selected structures in one of the frames of the time series of this region.

\subsection{Temporal variations of profiles}

To study the temporal behavior of the Ca II K line profile shapes, we have examined the line profiles at central
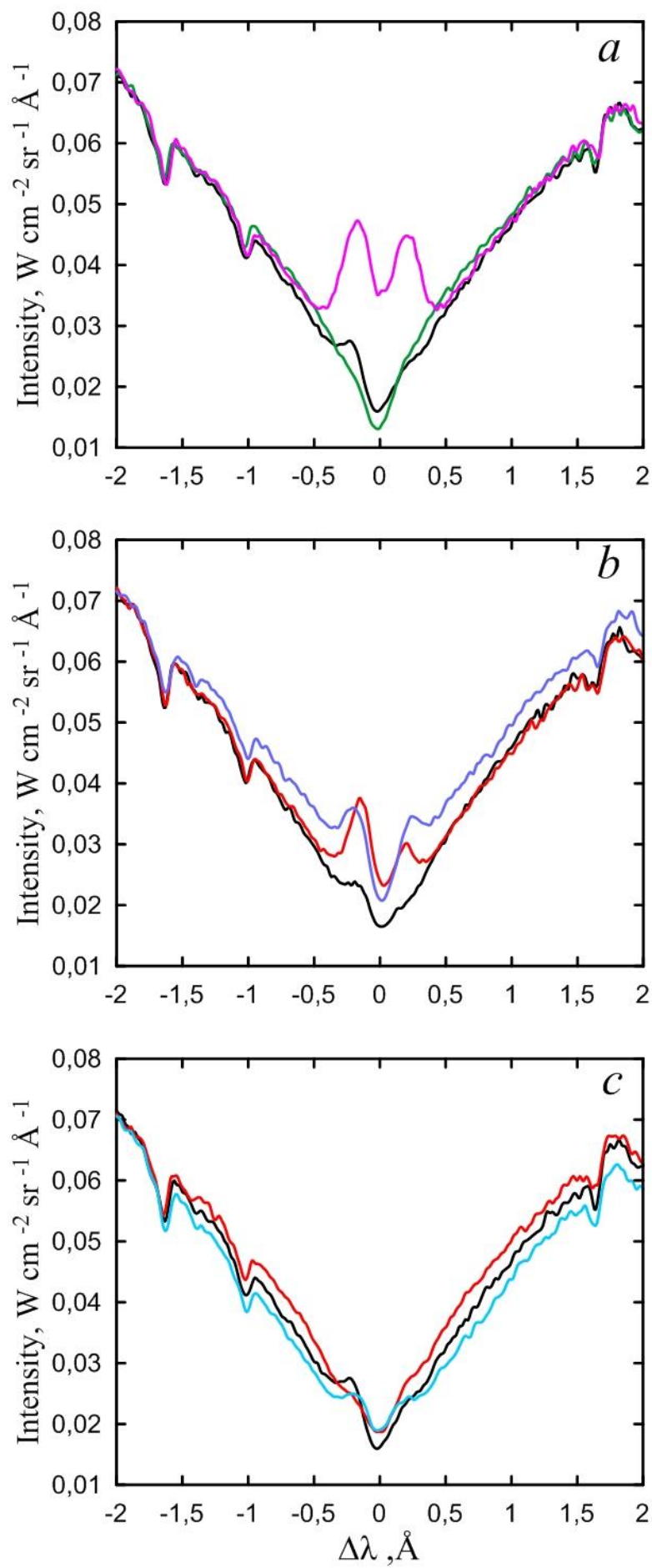

Figure 2. Ca II K line profiles in different chromospheric structures of S25W12 for one of the moments of time: $a$ is plage "b1" (purple), internetwork "c2" (black), area of low brightness "p1" (green); $b$ is network "n5" (blue), internetwork "c5" (black), network "n4" (red); $c$ is an area of intermediate brightness "x1" (blue), internetwork "c2" (black), network "n3" (red)

points of areas of all the selected chromospheric structures for each of the frames of the two time series. From these profiles we chose those with the largest $I_{\mathrm{K} 2 \mathrm{v}} / I_{\mathrm{K} 2 \mathrm{r}}>1.1 \div 1.15$ and smallest $I_{\mathrm{K} 2 \mathrm{v}} / I_{\mathrm{K} 2 \mathrm{r}}<0.85 \div 0.99$ intensity ratios. Examples of the profiles with measured shifts $\Delta \lambda_{\mathrm{K} 3}$ are given in Figure 3, $a-h$. It turned out that in most cases, at the moment of maximum brightening $I_{\mathrm{K} 2 \mathrm{v}}$ 

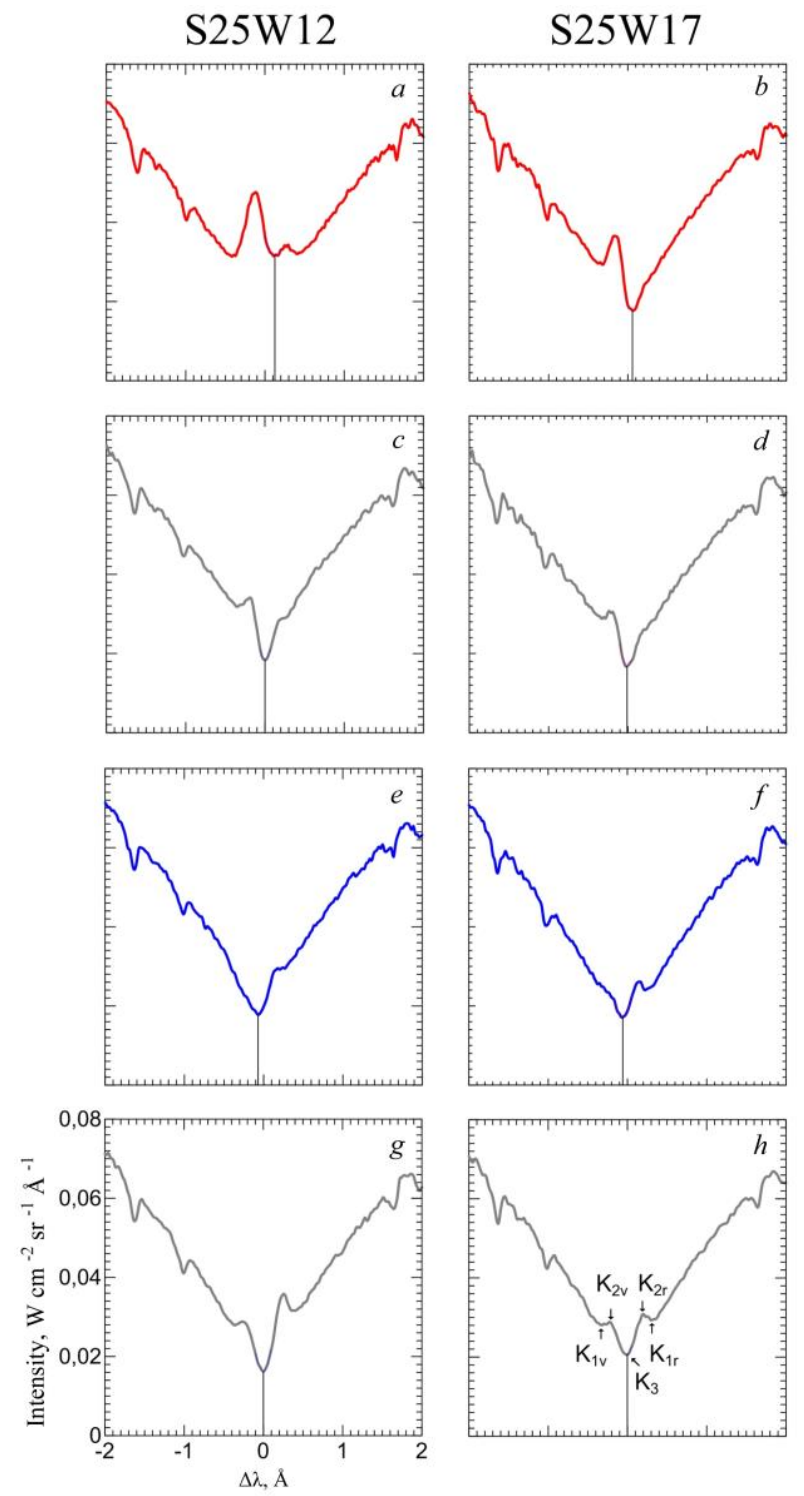

Figure 3. Variations of shifts of the $\mathrm{K}_{3}$ minimum in the $\mathrm{Ca}$ II K profiles in S25W12 (left panel) and S25W17 (right panel) when $I_{\mathrm{K} 2 \mathrm{v}} / I_{\mathrm{K} 2 \mathrm{r}}>1(a-d)$ and $I_{\mathrm{K} 2 \mathrm{v}} / I_{\mathrm{K} 2 \mathrm{r}}<1(e-h)$. Gray curves indicate cases of atypical behavior of the $\mathrm{K}_{2 \mathrm{v}}$-grain process. Positions of $K_{2 \mathrm{v}}, K_{2 \mathrm{r}}$ peaks, $\mathrm{K}_{1 \mathrm{v}}, \mathrm{K}_{1 \mathrm{r}}$, and $\mathrm{K}_{3}$ minima are shown

the $\mathrm{K}_{3}$ minimum demonstrates a red shift (Figure 3, $a, b$ ). This occurs in 45 of 48 cases for S25W12 and in 65 of 70 cases for $\mathrm{S} 25 \mathrm{~W} 17$. When $I_{\mathrm{K} 2 \mathrm{v}} / I_{\mathrm{K} 2 \mathrm{r}}$ becomes less than unity, the $\mathrm{K}_{3}$ minimum exhibits a blue shift (Figure 3,e,f) - 16 of 18 cases for S25W12 and 40 of 41 cases for S25W17.

Figure 4 gives examples of histograms of $\Delta \lambda_{\mathrm{K} 3}$ for $I_{\mathrm{K} 2 \mathrm{v}} / I_{\mathrm{K} 2 \mathrm{r}}>1.15$ and $I_{\mathrm{K} 2 \mathrm{v}} / I_{\mathrm{K} 2 \mathrm{r}}<0.85$. Statistical estimates of the shifts are listed in Table 1.

\subsection{Relationships between intensities and shifts of} the profile in the line center and wing

To investigate the relationships between different parameters of the $\mathrm{Ca}$ II $\mathrm{K}$ profiles in each chromospheric structure, we have used samples containing all spatial points belonging to the structure (e.g., all spatial points of all areas of the "c" structure) at all moments of time.
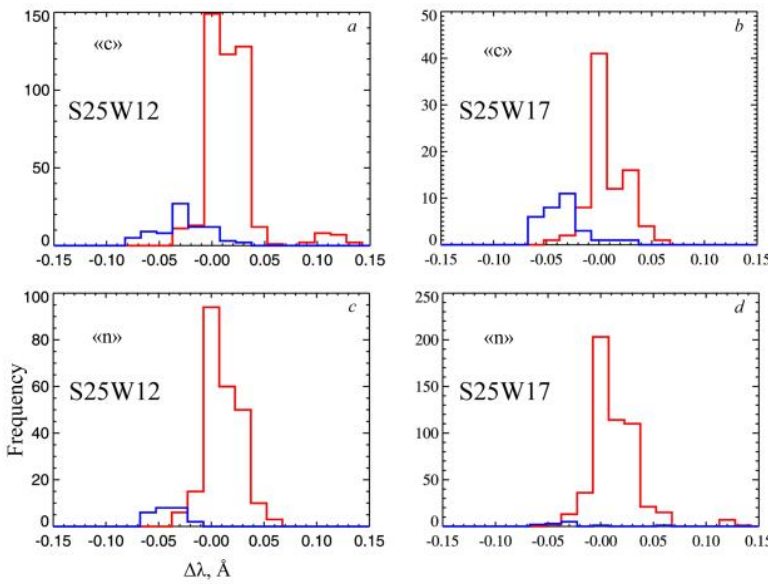

Figure 4. Histograms of line center shift $\Delta \lambda_{\mathrm{K} 3}$ for $I_{\mathrm{K} 2 \mathrm{v}} / I_{\mathrm{K} 2 \mathrm{r}}>1.15$ (red line) and $I_{\mathrm{K} 2 \mathrm{v}} / I_{\mathrm{K} 2 \mathrm{r}}<0.85$ (blue line) in two time series for the " $\mathrm{c}$ " and " $\mathrm{n}$ " structures

Table 2 presents calculated mean correlation coefficients between the selected Ca II K line parameters. In two top rows of the Table are coordinates of spatial regions, designations of chromospheric structures, and the number of points for each sample.

We also constructed scatter plots for all pairs of the parameters listed in Table 2. Figure 5 shows scatter plots for $\Delta \lambda_{\mathrm{K} 3}-I_{\mathrm{K} 2 \mathrm{v}} / I_{\mathrm{K} 2 \mathrm{r}}, \Delta \lambda_{\mathrm{K} 2 \mathrm{v}}-\Delta \lambda_{\mathrm{K} 2 \mathrm{r}}, I_{\mathrm{K} 2 \mathrm{r}}-I_{\mathrm{K} 2 \mathrm{v}}$, and $I_{\mathrm{K} 2 \mathrm{v}}-I_{\mathrm{K} 3}$.

Table 1

Statistical estimates of $\Delta \lambda_{\mathrm{K} 3}$

\begin{tabular}{|l|c|c|c|c|}
\hline \multirow{2}{*}{ Parameter } & \multicolumn{2}{|c|}{$I_{\mathrm{K} 2 \mathrm{v}} / I_{\mathrm{K} 2 \mathrm{r}}>1$} & \multicolumn{2}{c|}{$I_{\mathrm{K} 2 \mathrm{v}} / I_{\mathrm{K} 2 \mathrm{r}}<1$} \\
\cline { 2 - 5 } & $\begin{array}{c}\mathrm{S} 25 \\
\mathrm{~W} 12\end{array}$ & $\begin{array}{c}\mathrm{S} 25 \\
\mathrm{~W} 17\end{array}$ & $\begin{array}{c}\mathrm{S} 25 \\
\mathrm{~W} 12\end{array}$ & $\begin{array}{c}\mathrm{S} 25 \\
\mathrm{~W} 17\end{array}$ \\
\hline Number of points & 48 & 70 & 18 & 41 \\
\hline Minimum shift, $\AA$ & 0 & -0.01 & 0 & -0.008 \\
\hline Maximum shift, $\AA$ & 0.128 & 0.065 & -0.07 & -0.06 \\
\hline Range, $\AA$ & 0.128 & 0.075 & 0.07 & 0.052 \\
\hline Average shift, $\AA$ & 0.032 & 0.025 & -0.029 & -0.034 \\
\hline $\begin{array}{l}\text { Average } \\
\text { deviation, } \AA\end{array}$ & 0.015 & 0.012 & 0.013 & 0.011 \\
\hline
\end{tabular}

\section{DISCUSSION}

Despite the relatively modest set of our sample used for studying spatial variations of the profiles, it is easy to see features of the behavior of the Ca II K line intensity in different chromospheric structures. The profiles shown in Figure 2, $a-c$ allow us to compare intensity variations at the line core, in the $\mathrm{K}_{2}$ features, and line wing.

As noted in Section 2.1, the greatest differences between the profiles occur in their central parts. When comparing profiles for different structures in the wavelength range $\Delta \lambda \approx \pm 0.3 \AA$, we should pay attention to two characteristics - minimum intensity, $I_{\mathrm{K} 3}$, and profile shape. It is precisely the minimum intensity $I_{\mathrm{K} 3}$ which was used to identify the chromospheric structures in S25W12 and S25W17 (Figure 1, $a, b$ ). Despite $I_{\mathrm{K} 3}$ in individual profiles may slightly differ from the time-averaged 
Table 2

Mean correlation coefficients $\rho_{\mathrm{m}}$ for different pairs of parameters of Ca II K line profiles in different chromospheric structures

\begin{tabular}{|c|c|c|c|c|c|c|c|c|c|c|c|c|}
\hline \multirow[b]{2}{*}{ No. } & \multirow[t]{2}{*}{ Parameters } & \multicolumn{6}{|c|}{ S25W12 } & \multicolumn{5}{|c|}{ S25W17 } \\
\hline & & $\begin{array}{c}\text { "c" } \\
3375\end{array}$ & $\begin{array}{c}\text { "n" } \\
2205\end{array}$ & $\begin{array}{l}\text { "b" } \\
495\end{array}$ & $\begin{array}{c}\text { "f" } \\
2115\end{array}$ & $\begin{array}{l}\text { "x" } \\
2745\end{array}$ & $\begin{array}{c}\text { "p" } \\
1530\end{array}$ & $\begin{array}{c}\text { "c" } \\
1935\end{array}$ & $\begin{array}{c}\text { "n" } \\
2430\end{array}$ & $\begin{array}{l}\text { "b" } \\
495\end{array}$ & $\begin{array}{c}\text { "f" } \\
2160\end{array}$ & $\begin{array}{l}\text { "x" } \\
3195\end{array}$ \\
\hline 1 & $\Delta \lambda_{\mathrm{K} 2 \mathrm{v}}-\Delta \lambda_{\mathrm{K} 2 \mathrm{r}}$ & -0.08 & -0.17 & -0.02 & -0.11 & 0.04 & 0.02 & 0.14 & 0.18 & -0.11 & 0.03 & 0.18 \\
\hline 2 & $\Delta \lambda_{\mathrm{K} 2 \mathrm{r}}-I_{\mathrm{K} 2 \mathrm{r}}$ & 0.51 & 0.06 & 0.07 & 0.16 & 0.30 & 0.72 & 0.23 & 0.12 & 0.13 & -0.04 & 0.44 \\
\hline 3 & $\Delta \lambda_{\mathrm{K} 2 \mathrm{v}}-I_{\mathrm{K} 2 \mathrm{r}}$ & -0.33 & -0.17 & -0.20 & -0.12 & -0.08 & -0.01 & -0.11 & 0.07 & -0.17 & -0.03 & -0.09 \\
\hline 4 & $\Delta \lambda_{\mathrm{K} 2 \mathrm{r}}-I_{\mathrm{K} 2 \mathrm{v}}$ & 0.34 & 0.01 & 0.22 & 0.05 & 0.12 & 0.03 & 0.16 & 0.10 & 0.34 & 0.03 & 0.36 \\
\hline 5 & $\Delta \lambda_{\mathrm{K} 2 \mathrm{v}}-I_{\mathrm{K} 2 \mathrm{v}}$ & -0.20 & 0.26 & 0.06 & 0.09 & 0.07 & -0.71 & -0.01 & 0.27 & -0.11 & 0.20 & 0.14 \\
\hline 6 & $\Delta \lambda_{\mathrm{K} 2 \mathrm{r}}-\Delta \lambda_{\mathrm{K} 3}$ & 0.31 & 0.22 & 0.29 & 0.19 & 0.29 & 0.17 & 0.36 & 0.44 & 0.41 & 0.29 & 0.47 \\
\hline 7 & $\Delta \lambda_{\mathrm{K} 2 \mathrm{v}}-\Delta \lambda_{\mathrm{K} 3}$ & 0.25 & 0.29 & 0.34 & 0.10 & 0.10 & -0.21 & 0.46 & 0.68 & 0.14 & 0.51 & 0.54 \\
\hline 8 & $\Delta \lambda_{\mathrm{K} 3}-I_{\mathrm{K} 2 \mathrm{v}} / I_{\mathrm{K} 2 \mathrm{r}}$ & 0.46 & 0.59 & 0.70 & 0.58 & 0.63 & 0.17 & 0.54 & 0.46 & 0.70 & 0.66 & 0.58 \\
\hline 9 & $I_{\mathrm{K} 2 \mathrm{r}}-I_{\mathrm{K} 2 \mathrm{v}}$ & 0.66 & 0.49 & 0.29 & 0.51 & 0.48 & 0.25 & 0.71 & 0.77 & -0.03 & 0.66 & 0.57 \\
\hline 10 & $I_{\mathrm{K} 2 \mathrm{r}}-I_{\mathrm{K} 3}$ & 0.38 & 0.53 & 0.41 & 0.48 & 0.42 & 0.30 & 0.72 & 0.86 & 0.51 & 0.78 & 0.42 \\
\hline 11 & $I_{\mathrm{K} 2 \mathrm{v}}-I_{\mathrm{K} 3}$ & 0.35 & 0.53 & 0.63 & 0.41 & 0.49 & 0.33 & 0.68 & 0.82 & 0.20 & 0.70 & 0.39 \\
\hline 12 & $\left(\Delta \lambda_{\mathrm{K} 2 \mathrm{r}}-\Delta \lambda_{\mathrm{K} 2 \mathrm{v}}\right)-\Delta \lambda_{\mathrm{K} 2 \mathrm{v}}$ & -0.75 & -0.72 & -0.61 & -0.76 & -0.73 & -0.77 & -0.63 & -0.59 & -0.76 & -0.67 & -0.66 \\
\hline 13 & $\left(\Delta \lambda_{\mathrm{K} 2 \mathrm{r}}-\Delta \lambda_{\mathrm{K} 2 \mathrm{v}}\right)-\Delta \lambda_{\mathrm{K} 3}$ & 0.03 & -0.02 & 0.03 & 0.06 & 0.12 & 0.28 & -0.05 & -0.15 & 0.17 & -0.14 & -0.07 \\
\hline 14 & $\left(\Delta \lambda_{\mathrm{K} 2 \mathrm{r}}-\Delta \lambda_{\mathrm{K} 2 \mathrm{v}}\right)-I_{\mathrm{K} 2 \mathrm{v}}$ & 0.58 & -0.02 & 0.14 & 0.20 & 0.35 & 0.63 & 0.14 & -0.12 & 0.30 & -0.12 & 0.16 \\
\hline 15 & $\left(\Delta \lambda_{\mathrm{K} 2 \mathrm{r}}-\Delta \lambda_{\mathrm{K} 2 \mathrm{v}}\right)-I_{\mathrm{K} 3}$ & 0.06 & -0.33 & -0.33 & -0.23 & 0.05 & 0.11 & -0.03 & -0.27 & -0.24 & -0.29 & -0.17 \\
\hline 16 & $\left(\Delta \lambda_{\mathrm{K} 2 \mathrm{r}}-\Delta \lambda_{\mathrm{K} 2 \mathrm{v}}\right)-I_{\mathrm{K} 2 \mathrm{v}} / I_{\mathrm{K} 2 \mathrm{r}}$ & -0.18 & -0.26 & -0.01 & -0.13 & -0.08 & -0.15 & -0.14 & -0.28 & 0.13 & -0.16 & -0.15 \\
\hline 17 & $\Delta \lambda_{\mathrm{K} 2 \mathrm{av}}-\Delta \lambda_{\mathrm{K} 2 \mathrm{v}}$ & 0.70 & 0.58 & 0.59 & 0.68 & 0.76 & 0.78 & 0.74 & 0.74 & 0.69 & 0.70 & 0.78 \\
\hline 18 & $\Delta \lambda_{\mathrm{K} 2 \mathrm{ar}}-\Delta \lambda_{\mathrm{K} 3}$ & 0.41 & 0.39 & 0.44 & 0.21 & 0.26 & -0.06 & 0.54 & 0.72 & 0.41 & 0.55 & 0.66 \\
\hline 19 & $\Delta \lambda_{\mathrm{K} 2 \mathrm{av}}-I_{\mathrm{K} 2 \mathrm{v}}$ & -0.19 & -0.06 & 0.22 & -0.28 & -0.26 & -0.58 & 0.10 & 0.24 & 0.17 & 0.16 & 0.32 \\
\hline 20 & $\Delta \lambda_{\mathrm{K} 2 \mathrm{av}}-I_{\mathrm{K} 3}$ & -0.15 & -0.14 & -0.03 & -0.12 & -0.03 & -0.20 & 0.03 & 0.07 & -0.13 & -0.09 & 0.02 \\
\hline 21 & $\Delta \lambda_{\mathrm{K} 2 \mathrm{av}}-I_{\mathrm{K} 2 \mathrm{v}} / I_{\mathrm{K} 2 \mathrm{r}}$ & -0.14 & 0.17 & 0.26 & -0.04 & -0.11 & -0.72 & 0.02 & 0.15 & 0.16 & 0.25 & 0.21 \\
\hline
\end{tabular}

values presented in Figure 1, $a, b$, their variations remain similar in the spatial regions of interest.

The profile shape in the vicinity of the $\mathrm{K}$ line core and $\mathrm{K}_{2}$ features depends on physical conditions in the middle and lower chromosphere, where these features are formed. Of particular importance here is the set of such factors as temperature stratification and velocity field, which is determined by the dynamic state of the medium at a given moment of time in a given place. Moreover, the temperature stratification affects mainly the $\mathrm{K}_{2}$ features.

Special attention should be paid to the profile which is observed in the area of low brightness "p1". We have already written about profiles of this area in our previ- ous work [Grigoryeva et al., 2016], where we also discussed reversal-free profiles, which occurred in the "c" and " $x$ " structures. The reversal-free profiles have been examined in detail in [Rezaei et al., 2008]. According to these authors, such profiles comprise $\approx 25 \%$ of the number of the profiles they observed. To represent such a profile shape, they employed several 1D static models and the Holweger-Müller model [Holweger, Müller, 1974].

However, none of the hydrostatic models with chromospheric temperature increase the authors considered could reproduce the reversal-free profile shape.

As for intensities in line wings, here we faced the unexpected fact which is shown in Figure 2, $a, b$. Figure 2, $a$ displays the profiles that belong to the structures with 

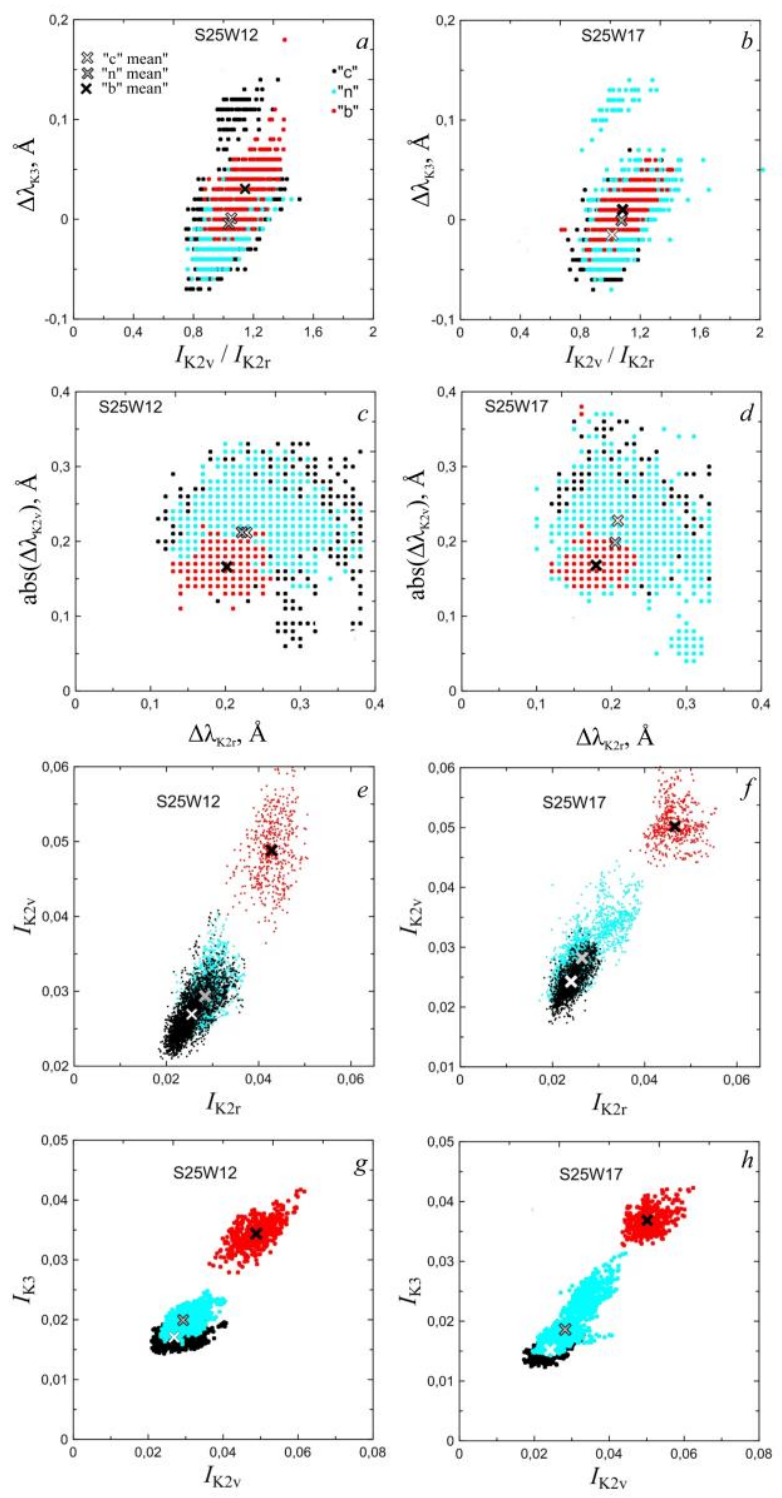

Figure 5. Scatter plots of different pairs of Ca II K profile parameters for "c", " $n$ ", and "b" structures in S25W12 (left panel) and S25W17 (right panel): $a, b$ are shifts of the line center $\Delta \lambda_{\mathrm{K} 3}$ versus the intensity ratio $I_{\mathrm{K} 2 \mathrm{v}} / I_{\mathrm{K} 2 \mathrm{r}} ; c, d$ are shifts of the violet emission peak $\Delta \lambda_{\mathrm{K} 2 \mathrm{v}}$ versus shifts of the red emission peak $\Delta \lambda_{\mathrm{K} 2 \mathrm{r}} ; e, f$ are intensities of the violet emission peak $I_{\mathrm{K} 2 \mathrm{v}}$ versus the intensity of the red emission peak $I_{\mathrm{K} 2 \mathrm{r}} ; g, h$ are in tensities of the $K_{3}$ minimum versus the intensity of the violet emission peak $I_{\mathrm{K} 2 \mathrm{v}}$. Black circles indicate "c" structures; blue circles, " $n$ " structures; red circles, "b" structures. The intensities are given in the same units as in Figure 3

extremely different physical conditions: plage "b1", internetwork "c2", and area with low brightness "p1". The intensities of the $\mathrm{K}$ line wings in these structures are almost similar as opposed to the intensities in their central parts. Such intensity variations begin at $\Delta \lambda= \pm 2 \AA$ and end near $\Delta \lambda$ values, which correspond to the location of the $\mathrm{K}_{1 \mathrm{v}}$ and $\mathrm{K}_{1 \mathrm{r}}$ features $(\Delta \lambda \approx \pm(0.3 \div 0.5 \AA))$. In Figure $2, b$, identical intensities are observed in the line wings for " $c 5$ " and " $\mathrm{n} 4$ ". A similar situation in the $\mathrm{Ca}$ II $\mathrm{H}$ line wing was observed by Beck et al. [2008]; the authors compared averaged profiles for a region with a weak magnetic field and for a region in which a magnetic field was not observed.
In Figure 2, $b$, the line profile in "n5" looks different. It is brighter than that in "c5" over the entire range $\Delta \lambda= \pm 2 \AA$. Moreover, it is brighter than that in " $n 4$ " in a part of the line wing from $\Delta \lambda= \pm 2 \AA$ to the location of the $\mathrm{K}_{1 \mathrm{v}}$ and $\mathrm{K}_{1 \mathrm{r}}$ features. Besides, in the line center the profile in " $\mathrm{n} 5$ " is darker and has more symmetrical $\mathrm{K}_{2}$ peaks than that in " $\mathrm{n} 4$ ". The significant difference in profile intensities for similar structures at the same moment of time can suggest either that they are at different stages of the dynamic process characteristic of structures of this type or that even in structures of one type different processes leading to the $\mathrm{Ca}$ II $\mathrm{K}$ line emission can work. If we compare the " 5 " and "c5" profiles and take into account that there are stronger magnetic fields in networks than in internetworks, for the brighter profile of "n5" we can use the assumption [Beck et al., 2008] about magnetic heating of the chromosphere. In this case, the line intensity increases in the spectral range involving the line wing. These authors do not, however, exclude a situation where the intensity increase in the line wing can be caused by a shift of the optical depth scale in the presence of a magnetic field rather than by the heating process.

The behavior of the $\mathrm{K}$ line intensities for the structures shown in Figure 2, $c$ is more relevant for " $\mathrm{n} 3$ " and "c2". Over the entire range $\Delta \lambda= \pm 2 \AA$, the network profile is brighter than the internetwork one, except for the $\mathrm{K}_{2 \mathrm{v}}$ peak, which is brighter in the internetwork that in the network. In the area of intermediate brightness " $\mathrm{x} 1$ ", the line wing is darker than that in "c2", although the line core is brighter.

From Figure 2, $a-c$ it follows that the behavior of the intensity in the $\mathrm{K}$ line wing, i.e. in the photosphere, does not always unambiguously indicate the type of emission that will occur in the chromosphere. It is seen that even with identical intensities in the line wing for individual structures the dramatic differences between profiles begin to appear at the level of formation of $\mathrm{K}_{1}$ areas, which is located between the upper photosphere and the lower chromosphere. Probably in these layers, the processes begin to occur which lead to the formation of the emission observed in the middle chromosphere.

The analysis of the $\mathrm{K}$ line profiles in different chromospheric structures of the same moment of time shows that their shape depends both on the temperature stratification and on the dynamic regime in the entire layer of the line formation - from the photosphere to the middle chromosphere.

One of the dynamic phenomena connected with temporal variations of $\mathrm{Ca}$ II line profiles are the socalled bright grains (flashes or points in other terminology), originally discovered inside the chromospheric network. In a number of studies [e.g., Cram, 1974; Cram et al., 1977; Cram, Dame, 1983; Rutten, Uitenbroek, 1991; Kamio, Kurokawa, 2006; Beck et al., 2008], the variations of Ca II line profile which are associated with bright grains are described as follows. A brightening occurs in line wings, which then extends to its center. At the same time, the line core gradually shifts to the red side. There is an intense brightening on the violet side from the line center with a substan- 
tial reduction or absence of the red emission peak on the red side, i.e. $I_{\mathrm{K} 2 \mathrm{v}} / I_{\mathrm{K} 2 \mathrm{r}}>1$. These brightenings are called $\mathrm{K}_{2 \mathrm{v}}$ grains. The $\mathrm{K}_{3}$ minimum at the moment of a $\mathrm{K}_{2 \mathrm{v}}$ flash is shifted at most to the red side. Then there is a sharp damping of the grain, and the $K_{3}$ minimum is shifted to the violet side. There are sometimes brightenings of the red emission peak $K_{2 r}$, i.e. $I_{\mathrm{K} 2 \mathrm{v}} / I_{\mathrm{K} 2 \mathrm{r}}<1$. The behavior of the line profile was called standard in the review [Rutten, Uitenbroek, 1991]. The size of the $\mathrm{K}_{2 \mathrm{v}}$ grain, according to different estimates, amounts to 0.7-3 arcsec [Liu, 1974; Beck et al., 2008; Vecchio et al., 2009]. The periodicity of $\mathrm{K}_{2 \mathrm{v}}$ grains was defined as $3 \mathrm{~min}$.

The process of generation of $\mathrm{K}_{2 \mathrm{v}}$ grains by weak acoustic shocks was simulated in [Carlsson, Stein, 1997]. A fairly close agreement has been found between the model and the behavior described above. In these years, other assumptions about physics of $\mathrm{K}_{2 \mathrm{v}}$ grains were published [see Kalkofen, 1996 and references therein].

Further observations revealed, however, that, first, the periodicity of occurrence of the grains may have not only a three-minute range [Kalkofen, 1996; Lites et al., 1999; Beck et al., 2008]. In our previous work [Grigoryeva et al., 2016], we have also shown that $K_{2 v}$ grains have a complex spectral composition of oscillations. Second, the line profile variation may differ from the standard one. And third, the grains may occur not only in the inner parts of the chromospheric network (internetwork), but also in the outer bright parts — network [Rutten, Uitenbroek, 1991; Kalkofen, 1996, 1997; Lites et al., 1999; Beck et al., 2008].

The study we have carried out has revealed that most shifts of $\mathrm{K}_{3}$ correspond to "standard" evolution of $I_{\mathrm{K} 2 \mathrm{v}}$ grains. We have also found deviations from the standard behavior. This can be seen in Figure $3, c, d, g, h$. As shown in Table 2, there are cases where the $\mathrm{K}_{3}$ minimum during a flash of $\mathrm{K}_{2 \mathrm{v}}$ grain appears to be shifted not to the red side but to the violet side ( 3 of 48 cases for S25W12 and 5 of 70 cases for S25W17). There are also cases of the red shift of the $\mathrm{K}_{3}$ minimum when $I_{\mathrm{K} 2 \mathrm{v}} / I_{\mathrm{K} 2 \mathrm{r}}$ is minimal. It should, however, be noted that for both the series in the " $n$ " and "c" structures the standard behavior prevails - the $\mathrm{K}_{3}$ line center shifts to the red side for large values of $I_{\mathrm{K} 2 \mathrm{v}} / I_{\mathrm{K} 2 \mathrm{r}}$ and to the violet side for its small values (Figure 4 ). The same tendency is also evident for the "f", " $x$ ", "b" structures (plots not shown here).

Also note that the number of profiles with $I_{\mathrm{K} 2 \mathrm{v}} / I_{\mathrm{K} 2 \mathrm{r}}<0.85$ is much less than the number of profiles with $I_{\mathrm{K} 2 \mathrm{v}} / I_{\mathrm{K} 2 \mathrm{r}}>1.15$ (Figure 4). The profiles with the intensity of the red emission peak $K_{2 r}$ higher than the intensity of the violet emission peak $\mathrm{K}_{2 \mathrm{v}}$ are rare. The positive asymmetry of the profiles $\left(I_{\mathrm{K} 2 \mathrm{v}} / I_{\mathrm{K} 2 \mathrm{r}}>1\right)$, as derived from model calculations [Bjørgen et al., 2017], is caused by the combination of an upward flow at a height of $1.2 \mathrm{Mm}$ and a downward flow at a height of $2.4 \mathrm{Mm}$, and by an increase in the source function at a height of $1.2 \mathrm{Mm}$.

According to observations obtained in earlier studies [see, e.g., Lites et al., 1993 and references therein], oscillatory processes in inner and outer parts of the chromospheric network differ. Lites et al. [1993] state that at the moment of a flash the network always exhibits sim- ultaneous brightening of violet and red emission peaks. Low-frequency oscillations $(\mathrm{f}<3 \mathrm{mHz}$ ) observed in the network, according to the data obtained by Lites et al. [1993], are not correlated with oscillations in lower layers and are bounded by the chromospheric layer, contrary to higher-frequency oscillations in the internetwork; From these observations Lites et al. [1993] conclude that oscillations in network and internetwork differ fundamentally. Observations [Vecchio et al., 2009] in the photospheric line Fe I $709.0 \mathrm{~nm}$ and the chromospheric line Ca II $854.2 \mathrm{~nm}$ with a high spatial resolution (0.166 "/pixel) have shown that purely acoustic three minute vertical oscillations are not ubiquitous. Even in the quiet internetwork, three minute oscillations occupy $25-30 \%$ of the area. They are significantly suppressed by the presence of tilted fibrils and smallerscale magnetic field elements. The magnetic field is largely responsible for the dynamical processes in the chromospheric network. Shocks with different characteristics occur both in network regions and in internetwork regions [Reardon et al., 2008].

In our previous work [Grigoryeva et al., 2016], we have studied in detail the occurrence of $\mathrm{K}_{2 \mathrm{v}}$ grains in various chromospheric structures. It turned out that the $\mathrm{K}_{2 \mathrm{v}}$ grains occurred in any of the structures we considered. Figure 3, $b$ of this paper, which exemplifies $\mathrm{K}_{2 \mathrm{v}}$ grains in the network, shows an apparent asymmetry of the intensities of the violet and red emission peaks during the maximum phase.

Our results suggest that there are a variety of oscillatory processes in the chromospheric network. This is likely to be largely due to the fact that the magnetic field affects not only the processes occurring in magnetic networks but also the processes occurring in internetworks.

To establish possible connections between the selected pairs of $\mathrm{K}$ line profile parameters (see Table 2), we constructed scatter plots. We used, as mentioned in Section 2.3, samples containing all spatial points of chromospheric structures of the same type at all moments of time of the time series. These plots represent general features of the structures. They are not directly related to different local dynamic processes occurring in the chromospheric network. Nevertheless, the plots of $\Delta \lambda_{\mathrm{K} 3}$ versus $I_{\mathrm{K} 2 \mathrm{v}} / I_{\mathrm{K} 2 \mathrm{r}}$ (Figure $5, a, b$ ) indicate the presence of the above tendency for the $K_{2 v}$ grains: $\Delta \lambda_{K 3}$ increases with increasing $I_{\mathrm{K} 2 \mathrm{v}} / I_{\mathrm{K} 2 \mathrm{r}}$, although there is a considerable dispersion of values. A high correlation between these parameters is observed in [Rezaei et al., 2007].

We may note a weak correlation or its absence between $\Delta \lambda_{\mathrm{K} 2 \mathrm{v}}$ and $\Delta \lambda_{\mathrm{K} 2 \mathrm{r}}$ (Table 2). This is also confirmed by the scatter plots for this pair of parameters (Figure 5, $c, d)$. The $\mathrm{K}_{2 \mathrm{v}}$ and $\mathrm{K}_{2 \mathrm{r}}$ peaks shift almost independently of each other. The absence of correlation can be partially explained by the fact that it is not always possible to determine the position of the $\mathrm{K}_{2 \mathrm{v}}$ peak. To an even greater degree this applies to the $\mathrm{K}_{2 \mathrm{r}}$ peak. However, without the problem of identifying peaks in plages, their shifts are not correlated either. In addition, in all the structures the intensities $I_{\mathrm{K} 2 \mathrm{v}}$ and $I_{\mathrm{K} 2 \mathrm{r}}$ with the same problems of identifying the position of the peaks have 
correlation coefficients significantly different from zero. This can also be seen in the scatter plots in Figure 5, $e, f$. An exception is the plage from S25W17 (see Table 2 and Figure 5, $f$ ).

We have found no significant correlation between $\Delta \lambda_{\mathrm{K} 2 \mathrm{r}}-\Delta \lambda_{\mathrm{K} 2 \mathrm{v}}$ and other parameters, with two exceptions. There is a clear correlation between $\Delta \lambda_{\mathrm{K} 2 \mathrm{r}}-\Delta \lambda_{\mathrm{K} 2 \mathrm{v}}$ and $\Delta \lambda_{\mathrm{K} 2 \mathrm{v}}$ and a correlation between $\Delta \lambda_{\mathrm{K} 2 \mathrm{r}}-\Delta \lambda_{\mathrm{K} 2 \mathrm{v}}$ and $I_{\mathrm{K} 2 \mathrm{v}}$ in the "c" and "p" structures with a weak magnetic field in S25W12.

There is a high correlation of $\Delta \lambda_{\mathrm{K} 2 \text { av }}$ with $\Delta \lambda_{\mathrm{K} 2 \mathrm{v}}$ and $\Delta \lambda_{\mathrm{K} 3}$. This parameter correlates weakly with $I_{\mathrm{K} 2 \mathrm{v}}, I_{\mathrm{K} 3}$ and $I_{\mathrm{K} 2 \mathrm{v}} / I_{\mathrm{K} 2 \mathrm{r}}$, except for the high correlation of $\Delta \lambda_{\mathrm{K} 2 \text { av }}$ with $I_{\mathrm{K} 2 \mathrm{v}}$ and $I_{\mathrm{K} 2 \mathrm{v}} / I_{\mathrm{K} 2 \mathrm{r}}$ for the "p" structure with a weak magnetic field.

There is a high correlation for the pairs of parameters $I_{\mathrm{K} 2 \mathrm{r}}-I_{\mathrm{K} 3}$ and $I_{\mathrm{K} 2 \mathrm{v}}-I_{\mathrm{K} 3}$ of S25W17. It is especially high for the "c" and " $n$ " structures. The intensity of the line profile in these structures tends to vary simultaneously in wings and in the center. In the plage, the correlation is lower. In S25W12, the correlation between the parameter pairs $I_{\mathrm{K} 2 \mathrm{r}}-I_{\mathrm{K} 3}$ and $I_{\mathrm{K} 2 \mathrm{v}}-I_{\mathrm{K} 3}$ is lower than in $\mathrm{S} 25 \mathrm{~W} 17$. The above tendencies can be seen in Figure 5, $f, h$, which presents the scatter plots of $I_{\mathrm{K} 3}$ versus $I_{\mathrm{K} 2 \mathrm{v}}$ for the two regions. A significant correlation between intensities in the $\mathrm{Ca}$ II $\mathrm{K}$ line core and wings was observed by Grossmann-Doerth et al. [1974]. The authors explained their findings by the source function distribution. The empirical source function is increased in a wide range of heights of the solar atmosphere, approximately from 600 to $2000 \mathrm{~km}$ (see Figure 8 in [Grossmann-Doerth et al., 1974]). This interpretation is also supported by results of 3D modeling [Bjørgen et al., 2017] (see their Figure $8, d$ ). The differences between the correlations in the two spatial regions, S25W17 and $\mathrm{S} 25 \mathrm{~W} 12$, for $I_{\mathrm{K} 2 \mathrm{r}}-I_{\mathrm{K} 3}$ and $I_{\mathrm{K} 2 \mathrm{v}}-I_{\mathrm{K} 3}$, formed in the lower and middle chromosphere, may be associated with different physical conditions in these regions. Recall that the S25W17 region is located at the base of the central part of the coronal hole, and the S25W12 region is located under the periphery of the bright coronal point near the coronal hole boundary (see Figure 1 in [Grigoryeva et al., 2016]). The relationship between the processes occurring at heights where $\mathrm{K}_{2 \mathrm{v}}\left(\mathrm{K}_{2 \mathrm{r}}\right)$ and $\mathrm{K}_{3}$ are formed may be weakened in case of possible locally closed magnetic topology [Vecchio et al., 2009] in S25W12.

\section{CONCLUSIONS}

1. In chromospheric structures of different types, observed at the same moment of time, there may be a situation where their $\mathrm{Ca}$ II $\mathrm{K}$ profiles are almost identical in the photosphere. The differences appear at the level of formation of the $\mathrm{K}_{1}$ feature, between the upper photosphere and the lower chromosphere. There are, however, structures for which the differences appear in the photosphere and continue in the chromosphere. This can be seen not only in different structures, but in structures of the same type as well. Probably, the characteristics of $\mathrm{Ca}$ II $\mathrm{K}$ profiles observed in different chromospheric structures are determined not only and not so much by the difference in the temperature stratification, but mainly by the nature of the dynamic process and by the magnetic field structure, which are present at a given time at a given point.

2. Temporal variations of Ca II K profiles suggest a variety of oscillations in the chromospheric network. $\mathrm{K}_{2 \mathrm{v}}$ grains occur in all the chromospheric structures we selected. The standard development of $\mathrm{K}_{2 \mathrm{v}}$ grains tends to dominate, although there are deviations from it.

3. Parameters of line profiles combined in terms of structures of the same type demonstrate a significant correlation between intensities in line center and wings, particularly for the "c" and " $\mathrm{n}$ " structures in S25W17, located at the base of the central part of the coronal hole. For the same structures from S25W12, correlations of $I_{\mathrm{K} 2 \mathrm{v}}$ and $I_{\mathrm{K} 2 \mathrm{r}}$ with $I_{\mathrm{K} 3}$ are lower. This may be associated with the location of the region at the base of the peripheral part of the bright coronal point near the coronal hole boundary.

We have found that the correlation between shifts of the $\mathrm{K}_{2 \mathrm{v}}$ and $\mathrm{K}_{2 \mathrm{r}}$ peaks for both the studied regions is weak or absent.

The results need further investigation involving observations of other regions on the Sun.

The work was performed with budgetary funding of Basic Research program II.16. The results were obtained using the equipment of Center for Common Use "Angara", http://ckp-rf.ru/ckp/3056.

We are grateful to V.M. Grigoryev for valuable discussions. We thank the referee for critical comments and constructive suggestions on the content of the manuscript.

\section{REFERENCES}

Bappu M.K.V., Sivaraman K.R. K emission-line widths and the solar chromosphere. Solar Phys. 1971, vol. 17, pp. 316-330.

Beck C., Schmidt W., Rezaei R., Rammacher W. The signature of chromospheric heating in Ca II $\mathrm{H}$ spectra. Astron. Astrophys. 2008, vol. 479, pp. 213-227. DOI: 10.1051/00046361:20078410.

Beck C., Khomenko E., Rezaei R., Collados M. The energy of waves in the photosphere and lower chromosphere I. Velocity statistics. Astron. Astrophys. 2009, vol. 507, pp. 453467. DOI: 10.1051/0004-6361/200911851.

Bjørgen J.P., Sukhorukov A.V., Leenaarts J., Carlsson M., de la Cruz Rodríguez, J., Scharmer G.B., Hansteen V.H. Three-dimensional modeling of the Ca II H\&K lines in the solar atmosphere. ArXiv:1712.01045v1 [astro-ph.SR]. 2017.

Carlsson M., Stein R.F. Formation of solar calcium H and K bright grains. Astrophys. J. 1997, vol. 481, pp. 500-514.

Cram L.E. High resolution spectroscopy of the disk chromospheres. Solar Phys. 1974, vol. 37, pp. 75-83.

Cram L.E., Damé L. High spatial and temporal resolution observations of the solar Ca II H line. Astrophys. J. 1983, vol. 272, pp. 355-361.

Cram L.E., Brown D.R., Beckers J.M. High resolution spectroscopy of the disk chromospheres. V. Space-time variations observed simultaneously in seven lines. Astron. Astrophys. 1977, vol. 57, pp. 211-220.

Fontenla J.M., Curdt W., Haberreiter M., Harder J., Tian H. Semiempirical models of the solar atmosphere. III. Set of nonLTE models for far-ultraviolet/extreme- ultraviolet irradiance computation. Astrophys. J. 2009, vol. 707, pp. 482-502.

Grigoryeva S.A., Turova I.P., Ozhogina O.A. Studying Ca II $\mathrm{K}$ line profile shapes and dynamic processes in the solar chromosphere at the base of a coronal hole. Solar Phys. 2016, 
vol. 291, pp. 1977-2002. DOI: 10.1007/s11207-016-0951-9.

Grossmann-Doerth U., Kneer F., von Uexküll M. Properties of the solar Ca II K-line at high spatial resolution. Solar Phys. 1974, vol. 37, pp. 85-94.

Holweger H., Müller E.A. The photospheric barium spectrum: solar abundance and collision broadening of Ba II lines by hydrogen. Solar Phys. 1974, vol. 39, pp. 19-30.

Kalkofen W. Chromospheric oscillations in $\mathrm{K}_{2 \mathrm{v}}$ bright points. Astrophys. J. 1996, vol. 468, L. 69-72.

Kalkofen W. Oscillations in chromospheric network bright points. Astrophys. J. 1997, vol. 486, L. 145-148.

Kamio S., Kurokawa H. The relation between Ca bright grains and oscillations in the photosphere. Astron. Astrophys. 2006, vol. 450, pp. 351-358. DOI: 10.1051/0004-6361:20054174.

Lites B.W., Rutten R.J., Kalkofen W. Dynamics of the solar chromospheres. I. Long-period network oscillations. Astrophys. J. 1993, vol. 414, pp. 345-356.

Lites B.W., Rutten R.J., Berger T.E. Dynamics of the solar chromospheres. II. Ca II $\mathrm{H} 2 \mathrm{v}$ and $\mathrm{K} 2 \mathrm{v}$ grains versus internetwork fields. Astrophys. J. 1999, vol. 517, pp. 1013-1033.

Liu S.-Y. Direct observational evidence for the propagation and dissipation of energy in the chromospheres. Astrophys. J. 1974, vol. 189, pp. 359-365.

Liu S.-Y., Skumanich A. An empirical interpretation for the time evolution of the Ca II line. Solar Phys. 1974, vol. 38, pp. 109-115.

Löfdahl M.G., Hillberg T., de la Cruz Rodriguez J., Vissers G., Scharmer G.B., Hagfors Haugan S.V., Fredvik T. A data-processing and metadata-generating pipeline for CHROMIS and CRISP // ArXiv:1804.03030v1 [astro-ph.IM] 2018, pp. 1-17.

Pasachoff J.M. Fine structure in Ca II on the solar disk. Solar Phys. 1970, vol. 12, pp. 202-215.
Reardon K.P., Lepreti F., Carbone V., Vecchio A. Evidence of shock-driven turbulence in the solar chromospheres. Astrophys. J. 2008, vol. 683, L. 207-210. DOI: 10.1086/591790.

Reardon K.P., Uitenbroek K., Cauzzi G. The solar chromospheres at high resolution with IBIS III. Comparison of Ca II K and Ca II $854.2 \mathrm{~nm}$ imaging. Astron. Astrophys. 2009, vol. 500, pp. 1239-1247. DOI: 10.1051/0004-6361:200811223.

Rezaei R., Schlichenmaier R., Beck C.A.R., Bruls J.H.M.J., Schmidt W. Relation between photospheric magnetic field and chromospheric emission. Astron. Astrophys. 2007, vol. 466, pp. 1131-1144. DOI: 10.1051/0004-6361:20067017.

Rezaei R., Bruls J.H.M.J., Schmidt W., Beck C., Kalkofen W., Schlichenmaier R. Reversal-free Ca II H profiles: a challenge for solar chromosphere modeling in quiet inter-network. Astron. Astrophys. 2008, vol. 484, pp. 503-509. DOI: 10.1051/00046361:20079050.

Rutten R.J., Uitenbroek H. Ca II $\mathrm{H}_{2 \mathrm{v}}$ and $\mathrm{K}_{2 \mathrm{v}}$ cell grains. Solar Phys. 1991, vol. 134, pp. 15-21.

Vecchio A., Cauzzi G., Reardon K.P. The solar chromospheres at high resolution with IBIS. II. Acoustic shocks in the quiet internetwork and the role of magnetic fields. Astron. Astrophys. 2009, vol. 494, pp. 269-286. DOI: 10.1051/00046361:200810694. 2018).

URL: http://ckp-angara.iszf.irk.ru (accessed March 20,

How to cite this article

Turova I.P., Grigoryeva S.A., Ozhogina O.A. Spatial and temporal variations of $\mathrm{Ca}$ II $\mathrm{K}$ line profile shapes in different structures of the solar chromosphere. I. Features of individual profiles. SolarTerrestrial Physics. 2018. Vol. 4. Iss. 4. P. 3-11. DOI: 10.12737/stp44201801. 